\title{
Electron spin and charge switching in a coupled quantum-dot-quantum ring system
}

\author{
B. Szafran, ${ }^{1,2}$ F. M. Peeters, ${ }^{1}$ and S. Bednarek ${ }^{2}$ \\ ${ }^{1}$ Departement Natuurkunde, Universiteit Antwerpen (Campus Drie Eiken), B-2610 Antwerpen, Belgium \\ ${ }^{2}$ Faculty of Physics and Nuclear Techniques, AGH University of Science and Technology, al. Mickiewicza 30, 30-059 Kraków, Poland
}

(Received 12 March 2004; revised manuscript received 20 May 2004; published 17 September 2004)

\begin{abstract}
Few-electron systems confined in a quantum dot laterally coupled to a surrounding quantum ring in the presence of an external magnetic field are studied by exact diagonalization. The distribution of electrons between the dot and the ring is influenced by the relative strength of the dot and ring confinement, and the magnetic field which induces transitions of electrons between the two parts of the system. These transitions are accompanied by changes in the periodicity of the Aharonov-Bohm oscillations of the ground-state angular momentum. The singlet-triplet splitting for a two electron system with one electron confined in the dot and the other in the ring exhibits piecewise linear dependence on the external field due to the Aharonov-Bohm effect for the ring-confined electron, in contrast to smooth oscillatory dependence of the exchange energy for laterally coupled dots in the side-by-side geometry.
\end{abstract}

DOI: 10.1103/PhysRevB.70.125310

PACS number(s): 73.21.La

\section{INTRODUCTION}

Coupling ${ }^{1-14}$ between semiconductor quantum dots ${ }^{15}$ results in the formation of so-called artificial molecules. Since most of the quantum dots have flat geometry, the coupling is realized either by vertical stacking ${ }^{1-5}$ or by fabrication of dots coupled laterally on the same plane ${ }^{6-14}$ Theoretical $^{6-11}$ considerations and experimental ${ }^{12-14}$ realizations of laterally coupled dots are based on the idea of dots placed side by side. This paper is devoted to few-electron states in an essentially different geometry of lateral coupling, namely, to a quantum dot surrounded by a quantum ring ${ }^{16}$ with a tunnel barrier separating both parts of the system. The confinement potential considered in this paper can be obtained using an atomic force microscope to locally oxidize ${ }^{17}$ the sample surface which results in the depletion of the two-dimensional electron gas (2DEG) underneath it. Alternatively one can apply split gates with a central cap gate surrounded by a thin collar gate on top of a planar $n \mathrm{AlGaAs}-\mathrm{GaAs}$ heterostructure containing a 2DEG. A proper geometry of split gates for the fabrication of the confinement potential considered in this paper was applied in the study ${ }^{18}$ of effects related to electron localization on local fluctuations of the confinement potential in the low electron density regime. The system studied in the present paper would require a sufficiently strong confinement which is less perturbed by fluctuations. The effect of local perturbations can be largely diminished by optimization ${ }^{19}$ of the size of electrodes for the strength of the electrostatic confinement potential.

Phase effects appearing in electron transport through quantum dots were studied in the Aharonov-Bohm interferometer. ${ }^{20,21}$ The potential geometry studied in this paper is a two-dimensional counterpart of quantum-dot quantum-well structures. ${ }^{22,23}$ Impurity effect on the singleelectron states in a three-dimensional quantum ring for strong in-plane confinement has been studied. ${ }^{24}$ Related to the present work is the magnetic coupling of a superconducting disk surrounded by a superconducting ring. ${ }^{25}$ In contrast to the work of Ref. 25, in the system considered here the coupling between the ring and the dot occurs through quan- tum mechanical tunnelling. The transfer of the charge between a quanum ring and inline- as well as side-coupled quantum dot and its effect on the persistent currents in mesoscopic samples was previously studed in the Andersonimpurity-type model. ${ }^{26}$

We study the effect of the magnetic field on the confined one-, two-, and three-electron systems using an exact diagonalization approach. In quantum dots and rings the magnetic field induces ground-state angular momentum transitions. However, the role of the electron-electron interaction for the transitions in these two structures is different. In quantum rings the interaction is of secondary ${ }^{27,28}$ importance for the angular momentum transitions which are mainly determined by the Aharonov-Bohm effect. In spinless ${ }^{27,29}$ few-electron systems the ground-state angular momentum is not influenced by the Coulomb interaction, and for electrons with spin the angular momentum of the ground state differs from the noninteracting case by at most $\hbar .^{28}$ On the other hand, in quantum dots the Coulomb interaction influences strongly the values of the magnetic field at which the angular momentum transitions appear. Moreover, in two- and three-electron systems these transitions are absent if there is no electronelectron interaction. In this paper we study the hybrid magnetic-field evolution of the electron spectra in the dot-inthe-ring geometry.

The magnetic-field along with the angular momentum transitions induces a redistribution of the electron charge in quantum dots. ${ }^{29,30}$ Here, we will show that in the considered geometry the magnetic field can be used to transfer the electrons from the dot to the ring or vice versa. We will also address the problem of the magnetic-field-induced trapping of electrons in local potential cavities. ${ }^{31}$

The spins of a pair of electrons localized in laterally coupled dots have been proposed ${ }^{6}$ as a possible realization of coupled qubits. A universal quantum gate requires the possibility of application of single-qubit as well as two-qubit rotations. For this purpose one should be able to address each of the electrons individually as well as to control the state of the pair, which requires the spatial separation of electrons and a tunable coupling between them. We studied the singlet- 
triplet splitting energy for the two-electron system with one electron localized in the dot and the other in the ring. We show that the angular momentum transitions, appearing for the ring-confined electrons as a consequence of the Aharonov-Bohm effect, lead to a simple piecewise linear dependence of the exchange energy on the external magnetic field. Since the unitary evolution in quantum computation needs precise control of the underlying qubit interaction this simple dependence makes our system a good candidate for the realization of the magnetic field controllable pair of spin qubits. Recently, it has been established ${ }^{32}$ that the spin relaxation time in quantum dots defined by electric gates in twodimensional electron gas is much longer than the qubit redout time in spin-to-charge conversion technique.

This papers is organized as follows: In the next section the present approach is explained, the single electron spectrum is described in Sec. III, the results for two and three electrons are given in Secs. IV and V, respectively, and Sec. VI contains the summary and conclusions.

\section{THEORY}

We study two-dimensional $\mathrm{N}$-electron systems confined in circular potentials using the effective mass Hamiltonian

$$
H=\sum_{i=1}^{N} h_{i}+\sum_{i=1}^{N} \sum_{j=i+1}^{N} \frac{e^{2}}{4 \pi \varepsilon \varepsilon_{0} r_{i j}}+B S_{z} g^{*} \mu_{B},
$$

where $\varepsilon$ is the dielectric constant, $g^{*}$ is the effective Landé factor, $\mu_{B}$ is the Bohr magneton, $S_{z}$ is the $z$ component of the total spin, $B$ is the magnetic field, and $h_{i}$ stands for the single-electron Hamiltonian, which written in the symmetric gauge $\mathbf{A}=(-B y / 2, B x / 2,0)$ has the form

$$
h=-\frac{\hbar^{2}}{2 m^{*}} \nabla^{2}+\frac{1}{8} m^{*} \omega_{c}^{2} \rho^{2}+\frac{1}{2} \hbar \omega_{c} l_{z}+V(\rho),
$$

with $m^{*}$ the electron effective mass, $l_{z}$ the $z$-component angular momentum operator, $\omega_{c}=e B / m^{*}$ the cyclotron frequency, and $V(\rho)$ the confinement potential. We adopt material parameters for GaAs, i.e., $\mathrm{m}^{*} / m_{0}=0.067, \epsilon=12.9$, and $g^{*}=-0.44$. The last term of Eq. (1), i.e., the spin Zeeman splitting energy is independent of the distribution of electrons between the different parts of the system as well as of the Coulomb interaction energy. Moreover, the value of the $g^{*}$ factor can be tuned by the admixtures of Al substituting $\mathrm{Ga}^{33}$ We have therefore decided to neglect the Zeeman effect in most of the results presented in this paper (unless explicitly stated otherwise).

We model a strictly two-dimensional cylindrically symmetric potential of a quantum dot placed within the quantum ring with the following confinement potential:

$$
V(\rho)=\min \left[m^{*} \omega_{i}^{2} \rho^{2} / 2+V_{0}, m^{*} \omega_{o}^{2}(\rho-R)^{2} / 2\right],
$$

where $\hbar \omega_{i}$ and $\hbar \omega_{o}$ are the confinement energies of the dot and the ring, respectively, and the radius of the ring $R$ is determined by the sum of oscillator lengths for the dot and ring potential and the barrier thickness $(b)$ according to formula $R=\sqrt{2 \hbar / m \omega_{i}}+\sqrt{2 \hbar / m \omega_{o}}+b$. This potential is parabolic

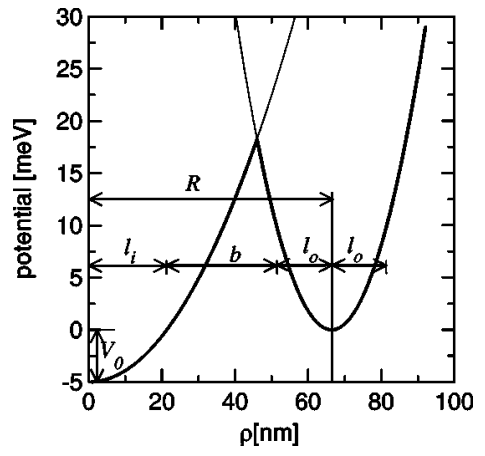

FIG. 1. Confinement potential [cf. Eq. (3) for $\hbar \omega_{i}=5 \mathrm{meV}$, $\hbar \omega_{o}=10 \mathrm{meV}, V_{0}=-5 \mathrm{meV}, b=30 \mathrm{~nm}$, and the GaAs effective mass $m^{*} / m_{0}=0.067$. The dot oscillator length $l_{i}=\sqrt{2 \hbar / m \omega_{i}}$ is equal to $21.33 \mathrm{~nm}$ and the oscillator length for the ring $l_{o}=15.08 \mathrm{~nm}$ which gives the ring radius $R=66.4 \mathrm{~nm}$.

within both the quantum dot and the quantum ring, $V_{0}$ is the depth of the dot confinement with respect to the bottom of the quantum ring potential. The confinement potential (3) is shown in Fig. 1 for $\hbar \omega_{i}=5 \mathrm{meV}, \hbar \omega_{o}=10 \mathrm{meV}, V_{0}$ $=-5 \mathrm{meV}$, and $b=30 \mathrm{~nm}$. A model potential parametrized similarly to Eq.(3) was used previously for the description $^{10,11}$ of side by side quantum dots. The cusp present in simple potentials of this type (cf. Fig. 1) is rather unphysical and cannot be realized in real structures, however this shortcoming is of secondary importance since the cusp appears in a region of space where the barrier potential is largest and the wave functions of the lowest energy levels are small. In the weak coupling limit (for large barrier thickness) approximate formulas for the dot- and ring-confined states as functions of the magnetic field can be given (see below).

In the present paper the single-electron eigenfunctions for Hamiltonian (2) and definite angular momentum are obtained numerically on a radial mesh of points using the finitedifference approach. Eigenstates of the two- and threeelectron Hamiltonian (1) are calculated using the standard configuration interaction method ${ }^{34}$ with a basis composed of Slater determinants built with single-electron wave functions. The Coulomb matrix elements are evaluated by a two-dimensional ${ }^{27}$ numerical integration. The few-electron states are described by the total spin $S$ and angular momentum $L$ quantum numbers. In this paper we discuss only the two- and three- electron states with nonpositive total angular momenta. We will therefore omit the minus sign for the angular momentum quantum number $L$.

\section{SINGLE-ELECTRON STATES}

The single-electron spectrum for $\hbar \omega_{i}=6 \mathrm{meV}, \hbar \omega_{o}$ $=11 \mathrm{meV}, V_{0}=0$, and $b=30 \mathrm{~nm}$ is shown in Fig. 2(a). For this relatively large barrier thickness, the low part of the energy spectrum is essentially a sum of the spectra of an electron localized in the dot and in the ring. The solid lines in Fig. 2 correspond to states localized in the ring and dashed lines to $s$ (lowest dashed line) and $p$ states localized in the dot. The ring part of the spectrum exhibits Aharonov-Bohm oscillations. The angular momentum of the lowest-energy ring-localized states takes on the subsequent values $0,-1$, 

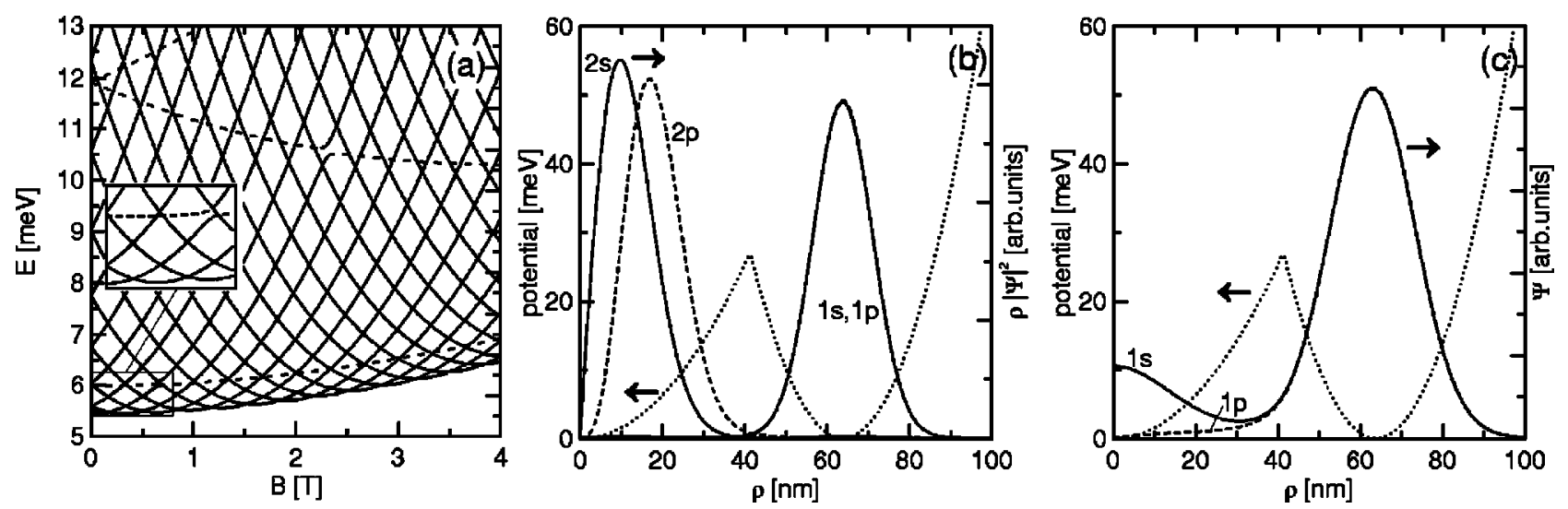

FIG. 2. Single-electron spectrum for $\hbar \omega_{i}=6 \mathrm{meV}, \hbar \omega_{o}=11 \mathrm{meV}, V_{0}=0$, and $b=30 \mathrm{~nm}(R=63.85 \mathrm{~nm})$. The solid lines correspond to states localized in the ring and the dashed lines to states localized in the dot. Lowest of the dashed lines corresponds to the $s$ state and the two higher to $p$ states. Inset shows the low-field and low-energy part of the spectrum-enlargement of the fragment surrounded by thin solid lines corresponding to anticrossing of 0 angular momentum dot- and ring-confined energy levels. Dotted lines in (b) and (c) shows the confinement potential (left scale) for the parameters applied in (a). Solid and dashed curves in (b) show the radial probability density $\rho|\psi|^{2}$ and in (c) the wave functions of the lowest states of $s$ and $p$ symmetry, respectively.

-2 , etc. [in $\hbar$ units] when the magnetic field increases. The period of these oscillations is $0.337 \mathrm{~T}$. This period corresponds to a flux quantum passing through a strictly onedimensional ring of radius $R_{1 \mathrm{D}}=62.51 \mathrm{~nm}$ which is in good agreement with the radius of the ring in the present model $R=63.85 \mathrm{~nm}$. The energy of the states localized in the dot change with the magnetic field more slowly than the energy of the ring-localized states. States with the same angular momentum change their order in anticrossings due to quantum mechanical tunnel coupling between the dot and the ring. Anticrossing for $s$ states appears for $B$ around $0.65 \mathrm{~T}$ [see inset of Fig. 2(a)]. A much wider anticrossing for $p$ states is visible around $2.4 \mathrm{~T}$.

Figure 2(b) shows the confinement potential for the parameters applied in Fig. 2(a) as well as the radial probability densities for the lowest $s$ - and $p$-symmetry states. The radial densities for the ring-localized states do not depend on the angular momentum. However, Fig. 2(c) shows that the $s$-wave function penetrates the dot region in a much stronger way than the $p$-type wave function. It will have an important consequence for the singlet-triplet splitting of the twoelectron states (see the next section). Note that the angular momentum has an opposite effect on the strength of the tunneling of the dot-localized states to the ring part of the potential. Barrier thickness is effectively smaller for the dotlocalized states of higher angular momentum [cf. Fig. 2(b)].

The dependence of the energy of the lowest dot-localized state can be very well approximated by the expression for the lowest Fock-Darwin state, i.e., $E_{\mathrm{dot}}=V_{0}+\sqrt{\left(\hbar \omega_{i}\right)^{2}+\left(\hbar \omega_{c} / 2\right)^{2}}$. Without the magnetic field the lowest energy ring-localized level is approximately equal to $\hbar \omega_{o} / 2$, i.e., to the energy of the single-dimensional harmonic oscillator in the radial direction. In the external field the envelope of the lowestenergy ring-localized level can be quite well approximated by $E_{\text {ring }}=\sqrt{\left(\hbar \omega_{o} / 2\right)^{2}+\left(\hbar \omega_{c} / 2\right)^{2}}$. These two formulas can be used in order to roughly determine whether the ground state of a single electron is localized in the dot or in the ring. For equal depth of the ring and the dot $\left(V_{0}=0\right)$ the magnetic field does not change the order of the lowest-energy dot- and ring- confined states. However, for $V_{0}=0$ and $\omega_{o} \approx 2 \omega_{i}$ the magnetic field can induce oscillations of the ground state localization from the dot to the ring, which results from the local deviations of the lowest ring-confined energy level from the smooth envelope [cf. Fig. 2(a)]. On the other hand, the magnetic field favors localization in the deep but small (thin) quantum dot (ring). This effect is illustrated in the following figure.

Figure 3 shows the energy spectrum for a $\hbar \omega_{i}$ which is increased with respect to Fig. 2 from 6 to $20 \mathrm{meV}$ and the bottom lowered by $V_{0}=-14 \mathrm{meV}$. For $B=0$ the low-energy part of the spectrum is the same as in the case shown in Fig. 2(a). However, the energy of the dot-localized state grows more slowly than the envelope of the ring-localized states. In consequence, the dot-localized state becomes the ground state for $B=3.3 \mathrm{~T}$. When the radius of the Landau orbit becomes smaller than the size of the local potential cavity, the electron can enter inside the dot without an extra increase of the kinetic energy due to the localization. Similar effects of trapping of electrons in local potential cavities at high magnetic fields are probably at the origin of the bunching of the charging lines observed in single-electron capacitance spec-

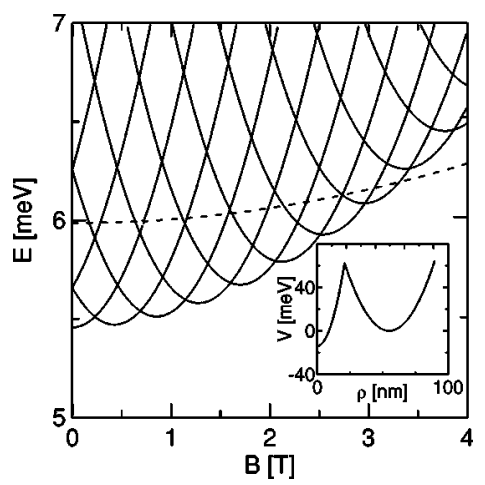

FIG. 3. Single-electron spectrum for $\hbar \omega_{i}=20 \mathrm{meV}, \hbar \omega_{o}$ $=11 \mathrm{meV}, V_{0}=-14 \mathrm{meV}$, and $d=30 \mathrm{~nm}$ (potential is plotted in the inset). The solid lines correspond to states localized in the ring and the dashed line to the lowest-energy state localized in the dot. 

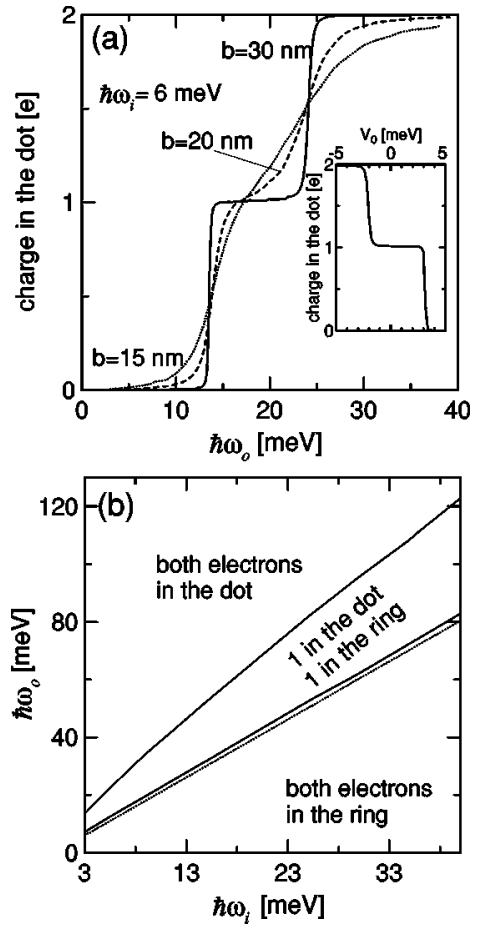

FIG. 4. Charge accumulated in the dot as a function of $\hbar \omega_{0}$ for different values of the barrier thickness and $V_{0}=0$. Inset in (a) shows the charge accumulated in the dot as function of $V_{0}$ for $b$ $=30 \mathrm{~nm}, \hbar \omega_{i}=6 \mathrm{meV}$, and $\hbar \omega_{o}=20 \mathrm{meV}$. (b) Phase diagram for the distribution of two electrons for $B=0, V_{0}=0$, and $b=30 \mathrm{~nm}$. Solid lines in (b) divide regions of different electron localization in the two-electron system. Above the dotted line the ground state of a single electron is localized within the dot.

troscopy of large quantum dots. ${ }^{31}$ The opposite effect, i.e., the change of the ground-state localization from the dot to the ring under influence of the external magnetic field is also possible if the ring is thin but with a bottom deeper than the dot.

\section{TWO ELECTRON SYSTEM}

For $B=0$ the ground state of the electron pair corresponds to zero total spin and angular momentum independently of the electron distribution between the two parts of the confinement potential. The electron distribution in the system can be illustrated by the charge accumulated within the dot. This quantity is calculated as the integral of the radial probability density from the origin to the cusp of the confinement potential (cf. Fig. 1). Figure 4(a) shows the dependence of the charge accumulated within the dot as a function of the ring oscillator energy for different barrier thickness, the dot confinement energy $\hbar \omega_{i}=6 \mathrm{meV}$ and equal depth of the dot and ring $\left(V_{0}=0\right)$. For $b=30 \mathrm{~nm}$ the dependence of the charge accumulated in the dot on the ring confinement energy is almost stepwise and it becomes smoothened for thinner barriers for which the separation of electrons between the two parts of the system is less distinct. The transition of electrons between the ring and the dot can also be provoked by changing the relative depth of the confinement potentials for fixed oscillator energies. This is illustrated in the inset to Fig. 4(a)
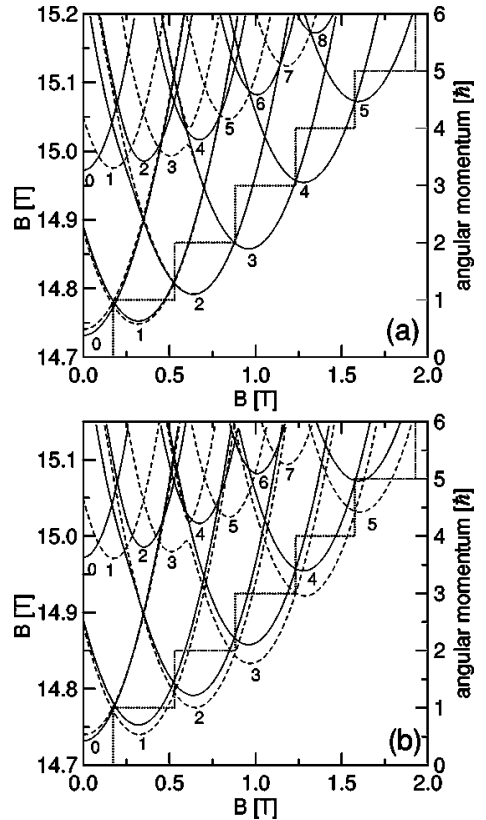

FIG. 5. Two-electron energy spectrum (left scale) for $b$ $=30 \mathrm{~nm}, V_{0}=0, \hbar \omega_{i}=6 \mathrm{meV}$, and $\hbar \omega_{o}=14 \mathrm{meV}$ (spin Zeeman effect neglected). Singlets (triplets) plotted with solid (dashed) lines. Numbers close to extrema of the lines denote the absolute values of the angular momentum in $\hbar$ units. The dotted line shows the absolute value of the angular momentum of the ground state of a single electron confined in the ring (right scale). (b) Same as (a) but with spin Zeeman effect included. Only the lowest energy level of the split spin triplet is plotted.

which shows the charge accumulated within the dot as a function of $V_{0}$ for the potential parameters $\hbar \omega_{i}=6 \mathrm{meV}$, $\hbar \omega_{o}=20 \mathrm{meV}, b=30 \mathrm{~nm}$, i.e., corresponding to the central plateau of the solid curve in the main part of Fig. 4(a).

Figure 4(b) shows the phase diagram for the spatial distribution of electrons in the two-electron system in the absence of the magnetic field for barrier thickness $b=30 \mathrm{~nm}$. Borders of regions corresponding to different electron distributions are marked with solid lines. Above the dotted line the ground state of a single electron is localized in the dot and below it-in the ring. The dotted line can be very well approximated by $\omega_{o}=2 \omega_{i}$, which is in agreement with the approximate formulas for the lowest-energy dot- and ringlocalized states given in the preceding section. In the noninteracting case this line would divide the regions in which both of the electrons are localized in the dot or in the well. In the presence of interaction a third region in which one of the electrons is localized in the dot and the other in the ring appears. This region of electron distribution starts slightly above the dotted line. This results from the fact that the Coulomb interaction, smallest for both electrons localized in the ring, stabilizes the ring-confined ground state for larger $\hbar \omega_{o}$ than in the noninteracting case. The central region of the phase diagram for which one electron resides in the dot and the other in the ring is particularly interesting from the point of view of potential spin quantum gate applications. $^{6}$

Let us now look at the magnetic field dependence of the two-electron energy spectrum for the potential parameters 


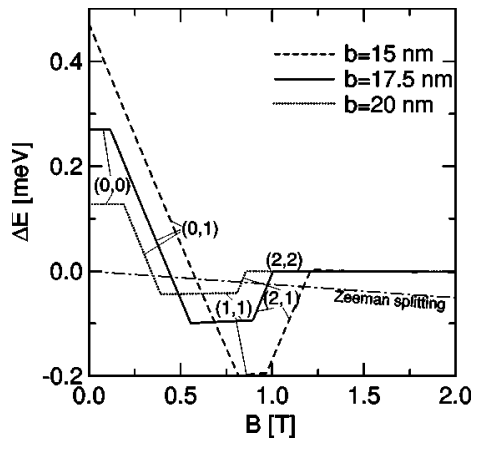

FIG. 6. Exchange energy, i.e., the energy difference of the lowest triplet and the lowest singlet energy levels for two electrons and $V_{0}=0, \hbar \omega_{i}=6 \mathrm{meV}$, and $\hbar \omega_{o}=18 \mathrm{meV}$ for different values of the barrier width and spin Zeeman splitting is neglected. The dashdotted line shows the Zeeman splitting between states with $S_{z}=0$ and $\hbar$.

corresponding to one electron in the dot and the other in the ring, i.e., for $V_{0}=0, b=30 \mathrm{~nm}, \hbar \omega_{i}=6 \mathrm{meV}$, and $\hbar \omega_{o}$ $=14 \mathrm{meV}$ presented in Fig. 5(a). For this potential the oneelectron ground state is localized in the dot. The angular momentum of the lowest excited ring-confined one-electron state is plotted with a dotted line (right scale). Comparison of this line with the ground-state energy crossings in the twoelectron spectrum shows that the angular momentum transitions in the two-electron system are due to the AharonovBohm effect for the electron confined within the ring. All the angular momentum of the system is therefore carried by the ring-confined electron while the electron confined in the dot remains in the $s$ state. Singlet-triplet splitting of the ground state [cf. the distance between the dashed and solid lines in Fig. 5(a)] disappears at larger angular momentum. This effect can be understood if we look back at Fig. 2(c) showing that the dot penetration of the ring-localized single-electron states decrease with their angular momentum. In Fig. 5(a) above $14.9 \mathrm{meV}$ the energy band corresponding to both electrons confined within the ring appears. Since in this band both ring-confined electrons are subject to the AharonovBohm effect the angular momentum of the lowest state in the band grows roughly twice ${ }^{28}$ as fast as in the ground state. The energy levels of even $L$ correspond to spin singlets and of odd $L$ to triplets. Around $0.6 \mathrm{~T}$ we observe an anticrossing of $L=3$ triplets corresponding to one and two electrons in the ring. The Zeeman effect [cf. Fig. 5(b)] for large $B$ lifts the ground-state degeneracy with respect to the spin.

The energy difference between the lowest spin singlet and triplet states, i.e., the exchange energy ${ }^{6}$ — an important quantity for the coupled spin qubit operations is also a very adequate measure of the strength of the tunnel coupling between the dot and the ring confined wave functions. Figure 6 shows the exchange energy (Zeeman energy neglected) for different values of the barrier thickness for $V_{0}=0, \hbar \omega_{i}$ $=6 \mathrm{meV}$, and $\hbar \omega_{o}=18 \mathrm{meV}$, i.e., for the central point of the plateau corresponding to one of the electrons localized in the dot [cf. Fig. 4(a)]. The exchange energy is nearly independent of magnetic field when the lowest singlet and the lowest triplet possess the same angular momentum and it distinctly decreases (grows) with the magnetic field when the $L$ of the

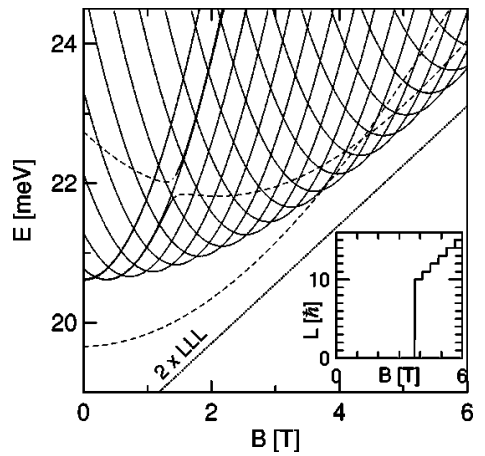

FIG. 7. Energy spectrum of two electrons for $b=30 \mathrm{~nm}, \hbar \omega_{i}$ $=6 \mathrm{meV}$, and $\hbar \omega_{o}=26 \mathrm{meV}$ (spin Zeeman effect neglected). The energy levels of states in which both (one) electrons are localized in the dot are plotted with dashed (solid) lines. The inset shows the ground state angular momentum. The dotted line corresponds to twice the lowest Landau level energy.

lowest triplet is larger (smaller) than $L$ of the lowest singlet. When the angular momentum of both singlet and triplet states exceed 2 , the exchange energy vanishes. The exchange energy is a piecewise linear function of the magnetic field in contrast to smooth oscillatory dependence of the exchange interaction on the magnetic field for side-by-side dots (cf. Fig. 4 of Ref. 11). In side-by-side dots the magnetic field induces a continuous decrease of the overlap of the wave functions of electrons confined in different dots. For the dot in the ring geometry this decrease is discontinuous due to the Aharonov-Bohm effect for the ring confined electron. Since the Aharonov-Bohm magnetic period is inversely proportional to the square of the ring radius one can largely reduce the range of the magnetic field in which the exchange energy is nonzero by a mere increase of $R$.

The magnetic field can change the distribution of the electrons between the coupled cavities. Consider the case of $b$ $=30 \mathrm{~nm}, V_{0}=0, \hbar \omega_{i}=6 \mathrm{meV}$ and $\hbar \omega_{o}=26 \mathrm{meV}$. For these parameters in the absence of the magnetic field both electrons are localized within the dot [cf. Fig. 4(b)], but the state corresponding to one electron in the ring is close in energy. Figure 7 shows the magnetic field dependence of the twoelectron energy spectrum for this potential. Energies of states corresponding to both electrons localized in the dot are plotted with dashed lines. The lower of these two energy levels is a spin singlet of $s$ symmetry. The upper dashed line corresponds to a spin triplet of $p$ symmetry, i.e., to the twoelectron maximum density droplet. ${ }^{35}$ Spin singlet of $p$-symmetry with both electrons localized in the dot lies higher in energy beyond the range presented in this figure. The energy levels plotted with solid lines correspond to one electron localized in the dot (in the lowest $s$ state) and the other in the ring. For $B=1.44 \mathrm{~T}$ an avoided crossings appears for the $L=1$ spin triplets. For $B=3.74 \mathrm{~T}$ the energy level of the dot localized state crosses the energy level of the state with $L=10$ corresponding to one electron in the dot and the other in the ring. Note that below $B=3.74 \mathrm{~T}$ in the groundstate the electrons are in the singlet state while above this field singlet and triplet states are nearly degenerate. Decoupling of spins, in the sense of vanishing exchange energy appears abruptly after crossing $B=3.74 \mathrm{~T}$. For $B=4.35 \mathrm{~T}$, a 


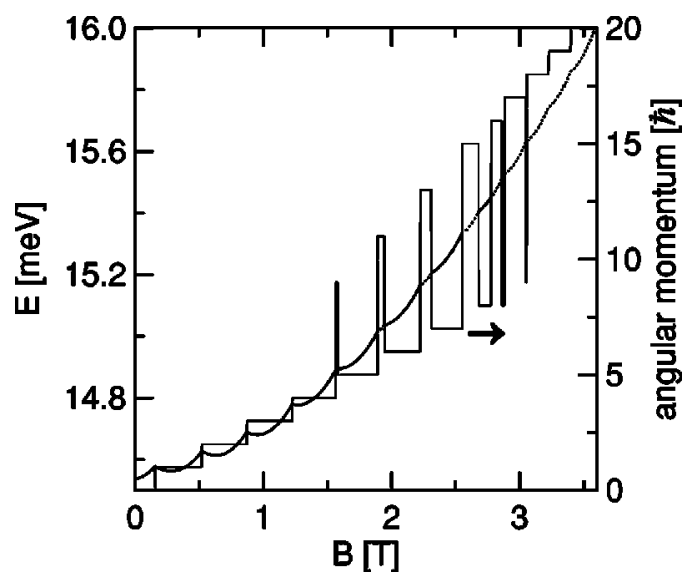

FIG. 8. Two-electron ground-state energy (left scale) for $b$ $=30 \mathrm{~nm}, V_{0}=0, \hbar \omega_{i}=6 \mathrm{meV}$, and $\hbar \omega_{o}=13.65 \mathrm{meV}$ ( spin Zeeman effect neglected). Energy of states corresponding to one electron in the dot and the other in the ring plotted with solid line. Energy of states in which both the electrons are localized in the ring are plotted by the dotted curve. The thin solid step-like line gives the total angular momentum which is referred to the right axis.

crossing of dot-localized singlet and triplet states appears. The dotted line shows twice the lowest Landau energy level. For $B>4 \mathrm{~T}$ the envelope of the energy levels with one electron in the dot and the other in the ring as well as the dot localized maximum density droplet run approximately parallel to the lowest Landau level (dotted line). Figure 7 shows that the magnetic field can change the electron occupation of the dot and the ring. Generally, for $V_{0}=0$ such an effect is not observed for a single electron. The appearance of this effect for two electrons is due to lowering of the Coulomb interaction energy when one of the electrons is transferred from the dot to the ring. Recently, ${ }^{36}$ it was shown that in the infinite magnetic field limit the ground-state electron distribution can be identified with the lowest energy configuration of classical $^{37}$ point charges. For $V_{0}=0$ the lowest-energy classical configuration corresponds to both electrons localized in the ring. One should therefore expect that at higher magnetic fields the second electron should also be transferred to the ring. However, the magnetic fields at which this effect could appear are beyond the reach of our numerical calculations.

For $V_{0}=0, b=30 \mathrm{~nm}, \hbar \omega_{i}=6 \mathrm{meV}$, and $\hbar \omega_{o}=13.65 \mathrm{meV}$ [the left end of the central plateau in the Fig. 4(b)] for $B$ $=0$ one of the electrons is localized in the dot and the other in the ring, but the state with two electrons localized in the ring is not much higher in energy. Figure 8 shows the ground-state energy and the ground-state angular momentum as functions of the magnetic field for this set of parameters. The state with one electron in the dot remains the ground state up to $1.6 \mathrm{~T}$. Between $B=1.6 \mathrm{~T}$ and $B=3.1 \mathrm{~T}$ the state with two electrons in the ring is almost degenerate with the state with one ring-confined electron and as a consequence the localization of the ground state changes several times as the magnetic field is increased. Ground-state ringlocalization becomes established above $3.1 \mathrm{~T}$. The period of the angular momentum transitions becomes halved with respect to the low magnetic fields, for which the ring is occupied by a single electron.

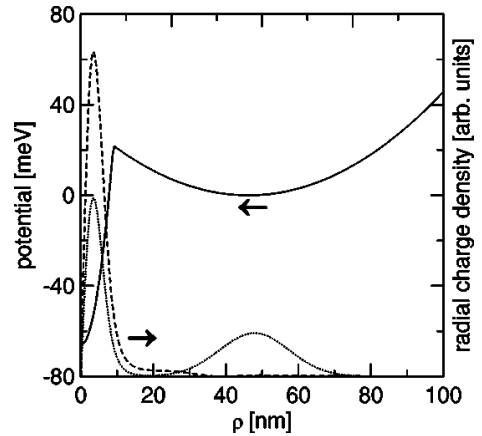

FIG. 9. Solid line (left scale): the external potential $\hbar \omega_{i}$ $=50 \mathrm{meV}$ and $\hbar \omega_{o}=6 \mathrm{meV}, b=20 \mathrm{~nm}, V_{0}=-66 \mathrm{meV}$. The ground state for $B=0$ corresponds to the $L=0$ singlet with one electron in the dot and the other in the ring. Radial density of this state plotted with dotted line (right scale). The first excited $s$ singlet state corresponds to both electrons in the dot (dashed line).

The magnetic field can induce opposite transitions of the electrons from the ring to the dot if the dot is small but deep. Consider the following set of parameters $\hbar \omega_{i}=50 \mathrm{meV}$, $\hbar \omega_{o}=6 \mathrm{meV}, b=20 \mathrm{~nm}$, and $V_{0}=-66 \mathrm{meV}$. Figure 9 shows the confinement potential and the radial probability density for the lowest two-electron singlet states with total angular momentum equal 0. For zero magnetic field in the groundstate one electron is localized in the ring and the other one in the dot. In the first excited $s$ singlet state both electrons reside within the dot. Note that in this case the ground state is more extended than the excited state as a consequence of the electron-electron interaction preventing the second electron from entering the dot. The magnetic field energy dependence is displayed in Fig. 10. The magnetic field has only a small influence on the energy of the singlet with both electrons localized in the dot. Around $B=2 \mathrm{~T}$, singlets corresponding to different electron distribution change their energy order with a pronounced anticrossing. For $B=5.725 \mathrm{~T}$ the dotlocalized singlet becomes the ground state. In this structure the Aharonov-Bohm oscillations are interrupted by the magnetic field which removes the second electron from the ring. As a consequence a giant singlet-triplet energy difference appears for $B>5.725 \mathrm{~T}$. This transition appears in spite of the Coulomb interaction energy which is increased when the second electron is trapped in the central cavity.

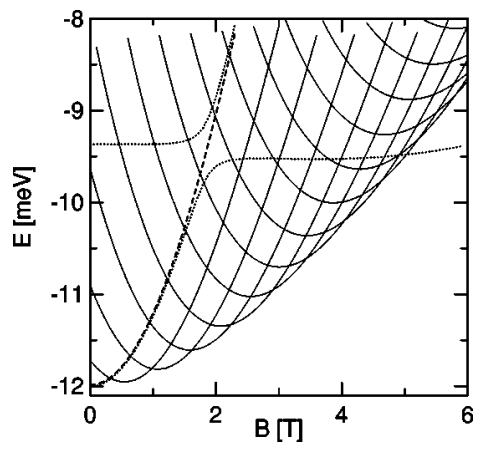

FIG. 10. Two-electron energy spectrum for the potential parameters of Fig. 9. Dotted lines show the energy levels of $s$ singlets. The dashed line corresponds to the $s$ triplet. 


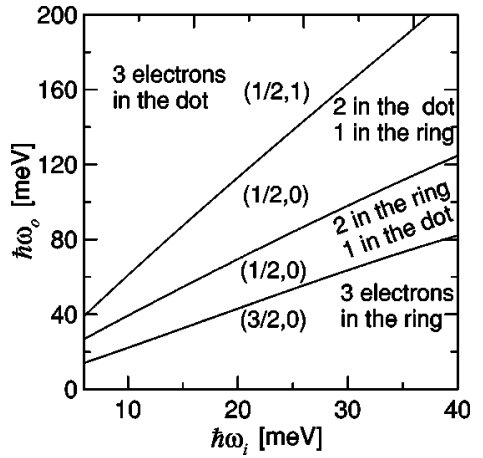

FIG. 11. Phase diagram for the electron distribution in the $\left(\hbar \omega_{i}\right.$, $\left.\hbar \omega_{o}\right)$ plane for $V_{0}=0$ and $b=30 \mathrm{~nm}$ in the absence of the magnetic field. Solid lines separate regions of different electron distributions. Numbers denote the ground-state total spin and total angular momentum quantum number $(S, L)$.

\section{THREE ELECTRONS}

Distribution of electrons in the three electron system without the magnetic field for $V_{0}=0$ and $b=30 \mathrm{~nm}$ is plotted in Fig. 11. Regions of different electron distribution are separated by the solid lines. For large dot confinement energy, i.e., $\omega_{i} \gg \omega_{o}$ all the electrons reside in the ring and the ground state corresponds to total spin $S=3 / 2$ and zero ${ }^{28}$ angular momentum. In the single-particle picture this state corresponds to electrons having parallel spin and occupying states with angular momentum $l=-1,0$, and 1 (in $\hbar$ units). For increasing ring confinement the electrons enter the dot one by one. In states with two electrons of opposite spins occupying the dot or the ring (cf. two central regions in Fig. 11 the ground state corresponds to $S=1 / 2$ and $L=0$. When the ring confinement energy is much larger (five times or more) than the dot confinement all the electrons occupy the lowest dot-confined energy levels forming the state of spin $S=1 / 2$ and angular momentum $L=1$.

In the preceding section we showed that for equal depth of the ring and the dot $\left(V_{0}=0\right)$ the electron-electron interaction triggered the magnetic-field-induced transitions of electrons from the dot to the ring. We found that in the threeelectron system the magnetic field can also induce the opposite transition from the ring to the dot. This is illustrated in Fig. 12 which shows the energy spectrum for $V_{0}=0, b$ $=30 \mathrm{~nm}, \hbar \omega_{i}=6 \mathrm{meV}$, and $\hbar \omega_{o}=37 \mathrm{meV}$. Solid lines in Fig. 12 show the energy levels corresponding to two electrons of opposite spins in the dot and one electron confined in the ring. All these states have $S=1 / 2$. Dashed lines correspond to spin-polarized states with $S=3 / 2$ in which the two dotconfined electrons occupy the $1 s$ and $1 p$ states. Energy levels corresponding to three electrons localized in the dot are shown by dotted curves. Quantum numbers of the dotconfined states are given in the figure. Thick solid steplike line at the bottom of the figure shows the absolute value of the ground-state angular momentum which is referred to the right axis. At $B=0$, the energy of the state in which all three electrons are localized in the dot with $L=1$ is $1 \mathrm{meV}$ higher in energy (cf. dotted line above $42 \mathrm{meV}$ ) above the ground state with two electrons in the dot and one in the ring. This energy level decreases initially with increasing magnetic

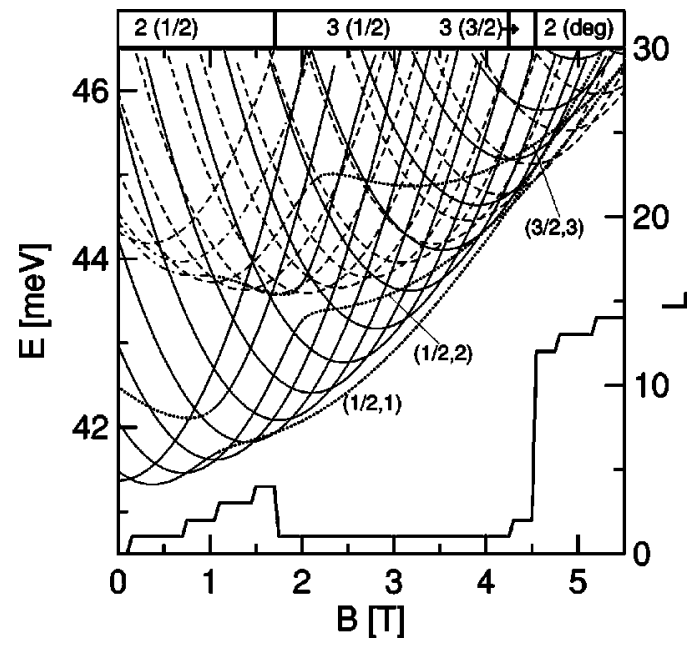

FIG. 12. Energy spectrum for $N=3, V_{0}=0, b=30 \mathrm{~nm}, \hbar \omega_{i}$ $=6 \mathrm{meV}$, and $\hbar \omega_{o}=37 \mathrm{meV}$. The solid (dashed) lines show the lowest energy levels with the two dot-confined electrons with $S=1 / 2$ and opposite ( $S=3 / 2$ and the same) spin and one electron in the ring. The states with the two dot-confined electrons of parallel spins are almost degenerate with respect to the spin orientation of the electron in the ring. The only exception is the state with $L=2$. The lower of the dash-dotted line shows this state for $S=1 / 2$ and the upper for $S=3 / 2$. Dotted lines correspond to all electrons confined in the dot. Quantum numbers $(L, S)$ of these states are indicated in the figure. Only nonpositive angular momenta are shown. The thick solid line in the lower part of the figure shows the the ground-state angular momentum quantum number (right scale). The panel above the upper axis shows the number of electrons in the $\operatorname{dot} n_{d}$ and $S$ for the ground state in format $n_{d}(S)$, "deg" stands for degeneracy of the $S=1 / 2$ and $3 / 2$ states.

field due to the interaction of the magnetic field with the magnetic momentum of the $p$ electron. This decrease results in an anticrossing of the $L=1$ energy levels corresponding to two and three dot-confined electrons around $B=1 \mathrm{~T}$. Another consequence of this anticrossing is a visibly increased region of $L=1$ ground-state stability between 0.15 and $0.7 \mathrm{~T}$. Subsequently for $B=1.7 \mathrm{~T}$, the state with three electrons in the dot and $L=1$ becomes the ground state. The transition of the third electron from the ring to the dot happens in spite of the electron-electron interaction which is not strong enough to prevent it.

For $B=0$, the energy of the lowest spin polarized state (cf. dashed lines) with $L=1$ is equal to about $44.25 \mathrm{meV}$. In this state the two electrons confined in the dot have the same spin and occupy $1 s$ and $1 p$ energy levels, while the ring-confined electron occupy the orbital with $l=0$. Note that level crossings appear at the same magnetic fields as in the lower branch with $S=1 / 2$ where two electrons are in the $1 s$ orbital confined in the dot (cf. solid lines in the Fig. 13). The angular momentum quantum number of these states is equal to the ring confined electron plus 1 - the angular momentum of the dot-confined subsystem. For the adopted large barrier thickness $b=30 \mathrm{~nm}$ the states of this band with $S=3 / 2$ are almost degenerate with $S=1 / 2$ states (omitted in the figure), i.e., the energy of the system is not influenced by the orientation of the spin of the ring-confined electron. The only exception appears for the $L=2$ state. The lower (upper) 


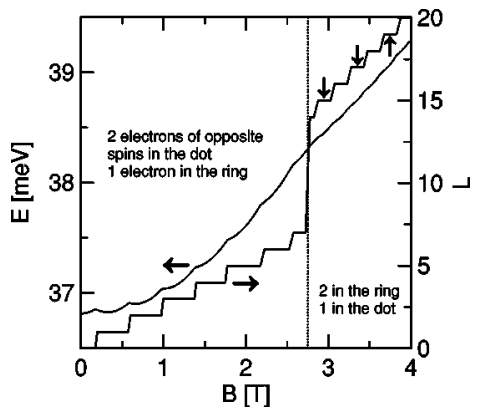

FIG. 13. Ground-state energy (left scale) and the absolute value of the ground-state angular momentum (right scale) for $N=3, V_{0}$ $=0, b=30 \mathrm{~nm}, \hbar \omega_{i}=6 \mathrm{meV}$, and $\hbar \omega_{o}=27 \mathrm{meV}$. The dotted vertical line marks the magnetic field for which the electron distribution is changed. The vertical arrows on the $L$ staircase correspond to triplet state of the ring subsystem.

dashed-dotted line shows the energy of the state with $L=2$ and $S=1 / 2(3 / 2)$. The reason of the lifted degeneracy is the fact that the energy of the state with $L=2$ and $S=1 / 2$ is pushed downward by the anticrossing with the dot-confined state of the same quantum numbers, similarly as the above discussed $L=1, S=1 / 2$ energy level in the lower part of the spectrum.

The angular momentum of the ground state with threeelectrons confined in the dot changes from 1 to 2 at $B$ $=4.3 \mathrm{~T}$ (cf. the crossing of the dotted lines). Above $4.5 \mathrm{~T}$ the ground state corresponds again to two electrons in the dot and one in the ring like for $B=0 \mathrm{~T}$, but now the dot-confined subsystem is spin-polarized (cf. the crossing of the dashed and dotted lines).

Figure 13 shows the ground-state energy for the same parameters as studied in Fig. 12 but with the ring confinement energy reduced from 37 to $27 \mathrm{meV}$. At $B=0$ the ground state still corresponds to two electrons in the dot and one in the ring, but the state with two ring-confined electrons is higher in energy by less than $1 \mathrm{meV}$. The envelope of the lowest energy level with one electron in the ring grows with the magnetic field faster than the envelope of the energy levels with two ring-confined electrons which results in the change of the ground-state electron distribution at $B$ $=2.75 \mathrm{~T}$. The dotted line in Fig. 12 marks the change in the electron distribution. Left of this line the ground state has $S=1 / 2$, the two dot-confined electrons are in the spin singlet, and the spin of the ring confined electron is arbitrary. Right of this line the spin-configuration of the ring-confined subsystem oscillates from singlet (for even $L$ ) to triplet (for odd $L)$ [cf. also the branch of ring confined electrons in Fig. 5(a)]. The magnetic fields for which the ring subsystem is in the triplet state have been marked by vertical arrows on the angular momentum staircase. The states with spin singlet of the ring subsystem correspond to $S=1 / 2$. Since the angular momenta of both ring-confined electrons exceed 6 the tunnel coupling between the dot and ring wave functions is negligible [cf. Fig. 8] and the spin of the dot confined electron does not influence the energy. Therefore, the states with triplet configuration of spins in the ring subsystem correspond to $S=1 / 2$ and $S=3 / 2$ degeneracy.

The envelope of the lowest $N=3$ energy levels with three electrons and two electrons in the ring run almost parallel to each other as a function of the external field. One should expect $^{36}$ that for equal depth of the ring and the dot at very large magnetic field the three-electron ground state corresponds to electrons forming an equilateral triangle in the ring, but in the studied magnetic field range we did not observe a distinct transition of the last electron from the dot to the ring.

\section{SUMMARY AND CONCLUSIONS}

We have considered a quantum dot inside a quantum ring-a unique example of lateral coupling realized with conservation of circular symmetry of the confinement potential. A simple model for the potential was used. The model assumes parabolic confinement in both the dot and the ring so approximate formulas can be given for the lowest-energy single-electron dot- and ring-confined states. One-, two-, and three-electron systems were studied using the exact diagonalization approach. We have investigated the distribution of electrons between the dot and the ring. This distribution depends not only on the parameters of the confinement potential but it can also be altered by an external magnetic field, which therefore can be used as a driving force to transfer the electrons from the dot to the ring or vice versa. The passage of an electron from the dot to the ring should be detectable by a change of the Aharonov-Bohm magnetic period. The present model also allows for simulation of the magnetic field induced electron trapping in local potential cavities. We have studied the exchange energy in the two electron system with one electron confined in the dot and the other in the ring. Due to the angular momentum transitions resulting from the Aharonov-Bohm effect for the ring-confined electron, the singlet-triplet splitting exhibits a piecewise linear dependence on the external magnetic field. This should be a more elegant method for the control of the spin-spin coupling than the smooth oscillatory dependence predicted for side-by-side coupled dots. ${ }^{11}$

\section{ACKNOWLEDGMENTS}

This paper has been partly supported by the Polish Ministry of Scientific Research and Information Technology in the framework of the solicited Grant No. PBZ-MIN-008/ P03/2003, the Flemish Science Foundation (FWO-Vl), the Concerted Action programme (IUAP), and the University of Antwerpen (VIS and GOA). One of us (B.S.) is supported by the Foundation for Polish Science (FNP). 
${ }^{1}$ J. J. Palacios and P. Hawrylak, Phys. Rev. B 51, 1769 (1995).

${ }^{2}$ B. Partoens and F. M. Peeters, Phys. Rev. Lett. 84, 4433 (2000).

${ }^{3}$ M. Pi, A. Emperador, M. Barranco, F. Garcias, K. Muraki, S. Tarucha, and D. G. Austing, Phys. Rev. Lett. 87, 066801 (2001).

${ }^{4}$ M. Bayer, P. Hawrylak, K. Hinzer, S. Fafard, M. Korkusinski, Z. R. Wasilewski, O. Stern, and A. Forchel, Science 291, 451 (2001).

${ }^{5}$ S. Bednarek, T. Chwiej, J. Adamowski, and B. Szafran, Phys. Rev. B 67, 205316 (2003).

${ }^{6}$ D. Loss and D. P. DiVincenzo, Phys. Rev. A 57, 120 (1998); G. Burkard, D. Loss, and D. P. DiVincenzo, Phys. Rev. B 59, 2070 (1999).

${ }^{7}$ C. Yannouleas and U. Landman, Phys. Rev. Lett. 82, 5325 (1999).

${ }^{8}$ S. Nagaraja, J.-P. Leburton, and R. M. Martin, Phys. Rev. B 60, 8759 (1999).

${ }^{9}$ J. Kohlemainen, S. M. Reimann, M. Koskinen, and M. Manninen, Eur. Phys. J. B 13, 731 (2000).

${ }^{10}$ A. Wensauer, O. Steffens, M. Suhrke, and U. Rössler, Phys. Rev. B 62, 2605 (2000).

${ }^{11}$ A. Harju, S. Siljamäki, and R. M. Nieminen, Phys. Rev. Lett. 88, 226804 (2002).

${ }^{12}$ F. R. Waugh, M. J. Berry, D. J. Mar, R. M. Westervelt, K. L. Campman, and A. C. Gossard, Phys. Rev. Lett. 75, 705 (1995).

${ }^{13}$ A. W. Holleitner, C. R. Decker, H. Qin, K. Eberl, and R. H. Blick, Phys. Rev. Lett. 87, 256802 (2001).

${ }^{14}$ J. M. Elzerman, R. Hanson, J. S. Greidanus, L. H. Willems van Beveren, S. De. Franceschi, L. M. K. Vandersypen, S. Tarucha, and L. P. Kouwenhoven, Phys. Rev. B 67, 161308 (2003).

${ }^{15}$ L. Jacak, P. Hawrylak, A. Wójs, Quantum Dots (Springer, Berlin, 1998)

${ }^{16}$ For a review see, S. Viefers, P. Koskinen, P. Singa Deo, and M. Manninen, Physica E (Amsterdam) 21, 1 (2004).

${ }^{17}$ A. Fuhrer, S. Lüscher, T. Ihn, T. Heinzel, K. Ensslin, W. Wegschelder, and M. Bichler, Nature (London) 413, 822 (2001).

${ }^{18}$ N. B. Zhitenev, M. Brodsky, R. C. Ashoori, L. N. Pfeiffer, and K. W. West, Science 285, 715 (1999).
${ }^{19}$ S. Bednarek, B. Szafran, K. Lis, and J. Adamowski, Phys. Rev. B 68, 155333 (2003).

${ }^{20}$ A. Yacoby, M. Heiblum, D. Mahalu, and Hadas Shtrikman, Phys. Rev. Lett. 74, 4047 (1995).

${ }^{21}$ K. Kobayashi, H. Aikawa, S. Katsumoto, and Y. Iye, Phys. Rev. B 68, 235304 (2003).

${ }^{22}$ D. Schooss, A. Mews, A. Eychmüller, and H. Weller, Phys. Rev. B 49, 17072 (1994).

${ }^{23}$ A. Mews, A. V. Kadavanich, U. Banin, and A. P. Alivisatos, Phys. Rev. B 53, R13242 (1996).

${ }^{24}$ B. S. Monozon and P. Schmelcher, Phys. Rev. B 67, 045203 (2003).

${ }^{25}$ B. J. Baelus, S. V. Yampolskii, and F. M. Peeters, Phys. Rev. B 66, 024517 (2002).

${ }^{26}$ M Büttiker and C. A. Stafford, Phys. Rev. Lett. 76495 (1996).

${ }^{27}$ T. Chakraborty and P. Pietiläinen, Phys. Rev. B 50, 8460 (1994).

${ }^{28}$ K. Niemielä, P. Pietiläinen, P. Hyvönen, and T. Chakraborty, Europhys. Lett. 36, 533 (1996).

${ }^{29}$ S. M. Reimann and M. Manninen, Rev. Mod. Phys. 74, 1283 (2002).

${ }^{30}$ B. Szafran, S. Bednarek, and J. Adamowski, Phys. Rev. B 67, 045311 (2003); J. Phys.: Condens. Matter 15, 4189 (2003).

${ }^{31}$ N. B. Zhitenev, R. C. Ashoori, L. N. Pfeiffer, and K. W. West, Phys. Rev. Lett. 79, 2308 (1997).

${ }^{32}$ R. Hanson, B. Witkamp, L. M. K. Vandersypen, L. H. Willems van Beven, J. M. Elzerman, and L. P. Kouwenhoven, Phys. Rev. Lett. 91, 196802 (2003).

${ }^{33}$ K. Ensslin, D. C. Driscoll, A. C. Gossard, and D. D. Awschalom, Nature (London) 414, 619 (2001).

${ }^{34}$ B. Szafran, S. Bednarek, J. Adamowski, M. Tavernier, E. Anisimovas, and F. M. Peeters, Eur. Phys. J. D 28, 373 (2004).

${ }^{35}$ A. H. MacDonald, S. R. E. Yang, and M. D. Johnson, Aust. J. Phys. 46, 345 (1993).

${ }^{36}$ B. Szafran, F. M. Peeters, S. Bednarek, and J. Adamowski, Phys. Rev. B 69, 125344 (2004).

${ }^{37}$ V. M. Bedanov and F. M. Peeters, Phys. Rev. B 49, 2667 (1994). 